\title{
Magnetization behavior of ferrofluids with cryogenically imaged dipolar chains
}

\author{
M Klokkenburg ${ }^{1}$, B H Erné ${ }^{1}$, V Mendelev ${ }^{2}$ and A O Ivanov ${ }^{2}$ \\ ${ }^{1}$ Van 't Hoff Laboratory for Physical and Colloid Chemistry, Science Faculty, \\ Utrecht University, Padualaan 8, 3584 CH Utrecht, The Netherlands \\ ${ }^{2}$ Department of Mathematical Physics, Urals State University, Lenin Avenue 51, \\ 620083 Ekaterinburg, Russian Federation
}

E-mail: M.Klokkenburg@chem.uu.nl, B.H.Erne@uu.nl and Alexey.Ivanov@usu.ru

Received 1 April 2008

Published 1 May 2008

Online at stacks.iop.org/JPhysCM/20/204113

\begin{abstract}
Theories and simulations have demonstrated that field-induced dipolar chains affect the static magnetic properties of ferrofluids. Experimental verification, however, has been complicated by the high polydispersity of the available ferrofluids, and the morphology of the dipolar chains was left to the imagination. We now present the concentration- and field-dependent magnetization of particularly well-defined ferrofluids, with a low polydispersity, three different average particle sizes, and with dipolar chains that were imaged with and without magnetic field using cryogenic transmission electron microscopy. At low concentrations, the magnetization curves obey the Langevin equation for noninteracting dipoles. Magnetization curves for the largest particles strongly deviate from the Langevin equation but quantitatively agree with a recently developed mean-field model that incorporates the field-dependent formation and alignment of flexible dipolar chains. The combination of magnetic results and in situ electron microscopy images provides original new evidence for the effect of dipolar chains on the field-dependent magnetization of ferrofluids.
\end{abstract}

(Some figures in this article are in colour only in the electronic version)

\section{Introduction}

The dielectric response of dipolar fluids not only depends on the interaction of individual dipoles with the external field but also on the interactions between the dipoles. Describing the dipolar interactions theoretically is a long-standing problem. When the dipole-dipole interactions exceed the thermal energy $k_{\mathrm{B}} T$, the classical theories of Langevin [1], Weiss [2], Debye [3], and Onsager [4] strongly differ in their predictions at high concentrations. Much work has been done to develop improved theoretical descriptions, and evaluating them is a task on its own (for a review see [5]). An experimental approach for evaluating the theories is fitting them to magnetization measurements on so-called ferrofluids, colloidal dispersions of single-domain magnetic nanoparticles. Until now, a drawback of the ferrofluids investigated has been that they are highly polydisperse, which complicates quantitative comparison with theory [6]. Moreover, even though experimental evidence for field-induced dipolar chaining in ferrofluids was found from small-angle scattering, magneto-optical effects, and other techniques [7], the interpretation of the results was model dependent, and the morphology of the dipolar chains was left to the imagination.

Here, we perform field- and concentration-dependent magnetization measurements on weakly polydisperse, singledomain magnetite $\left(\mathrm{Fe}_{3} \mathrm{O}_{4}\right)$ colloids, whose dipolar structures were previously demonstrated and quantified in zero and applied magnetic field by cryogenic transmission electron microscopy [8-10], and we show that the recently developed flexible chain model $[11,12]$ provides a quantitative description of the magnetization properties of these ferrofluids up to concentrations of $7 \%$.

\section{Experimental details}

Magnetization curves were measured for model ferrofluids whose preparation and properties have been described in detail elsewhere [8-10, 13, 14]. In brief, the fluids consist of weakly polydisperse magnetite nanoparticles with 
Table 1. Sample characteristics of systems A, B, and C.

\begin{tabular}{lrlcl}
\hline Code & $d_{\text {TEM }}(\mathrm{nm})$ & $m\left(10^{-20} \mathrm{~A} \mathrm{~m}^{2}\right)$ & $d_{\mathrm{M}}(\mathrm{nm})$ & $\lambda$ \\
\hline $\mathrm{A}$ & $21.0 \pm 2.4$ & 162 & 18.6 & 4.4 \\
$\mathrm{~B}$ & $16.1 \pm 2.6$ & 64.7 & 13.7 & 1.7 \\
$\mathrm{C}$ & $9.6 \pm 1.2$ & 18.6 & 9.0 & 0.5 \\
\hline
\end{tabular}

an oleic acid surface layer and dispersed in the solvent Decalin. Dilutions were done with a $1 \mathrm{mM}$ solution of oleic acid in Decalin to prevent surfactant desorption [14]. Three systems with different average particle diameters are examined, corresponding to three different dipole-dipole coupling parameters (see table 1). The diameters $d_{\text {TEM }}$ are from transmission electron microscopy [8-10].

The dipole moments $m$ in table 1 are obtained from the concentration dependence of the so-called initial (low-field) magnetic susceptibility in the limit of low concentrations, where the dipole-dipole interactions are negligible. The initial susceptibility then corresponds to the linear low-field part of the Langevin equation,

$$
M_{\mathrm{L}}=M_{\mathrm{sat}} L(\alpha)=M_{\mathrm{sat}}\left(\operatorname{coth}(\alpha)-\frac{1}{\alpha}\right)
$$

where $M_{\mathrm{L}}$ is the sample magnetization in $\mathrm{A} \mathrm{m}^{-1}$ or $\mathrm{G}, M_{\text {sat }}$ the saturation magnetization, $L$ is the Langevin function, and $\alpha=\mu_{0} m H /\left(k_{\mathrm{B}} T\right)$, with $\mu_{0}=4 \pi \times 10^{-7} \mathrm{~J} \mathrm{~A}^{-2} \mathrm{~m}^{-1}$ and $H$ the magnetic field in $\mathrm{A} \mathrm{m}^{-1}$. In the low-field limit, the Langevin magnetic susceptibility $\chi_{\mathrm{L}}=M_{\mathrm{L}} / H$ is linear in the particle number density $n$ :

$$
\chi_{\mathrm{L}}=\frac{\mu_{0} n m^{2}}{3 k_{\mathrm{B}} T}=8 \lambda \phi
$$

where $\phi$ is the particle volume fraction, defined as the total volume of particles, including the oleic acid layer, divided by the sample volume, and $\lambda=\mu_{0} m^{2} /\left(4 \pi k_{\mathrm{B}} T \sigma^{3}\right)$ is the magnetic coupling constant, with $\sigma$ the hard core diameter of the particles. The values for $\lambda$ in table 1 assume that the outer diameter $\sigma$ of the particles is equal to the core diameter $d_{\mathrm{TEM}}$ plus a $2 \mathrm{~nm}$ oleic acid layer [14]. The magnetic diameters $d_{\mathrm{M}}$ in table 1 are calculated from $m$ using a bulk magnetization of $480 \mathrm{kA} \mathrm{m}^{-1}$ for magnetite $\left(\mathrm{Fe}_{3} \mathrm{O}_{4}\right)$ [15]. Comparison of $d_{\text {TEM }}$ with $d_{\mathrm{M}}$ suggests the presence of a magnetically disordered iron oxide surface layer [16] of $1.2 \mathrm{~nm}$ for systems A and B, and $0.3 \mathrm{~nm}$ for system $\mathrm{C}$. In the light of the $1 \mathrm{~nm}$ resolution of TEM, the conclusion is that the particles can be modeled as bulk magnetite spheres and that the particles are so large that surface effects can be neglected in our analysis of the data.

Magnetization measurements were conducted on a MicroMag 2900 alternating gradient magnetometer from Princeton Measurements Corporation. The principle and performance of practically the same apparatus were described by Flanders [17]. The AC field was kept low $\left(1 \mathrm{kA} \mathrm{m}^{-1}\right)$ to avoid nonlinear effects on the measurements [18] (AC fields of $100 \mathrm{~A} \mathrm{~m}^{-1}$ gave practically the same results with much more noise). Dispersions of the nanoparticles were contained in glass cups of dimensions $4 \mathrm{~mm} \times 4 \mathrm{~mm} \times 0.4 \mathrm{~mm}$, with the

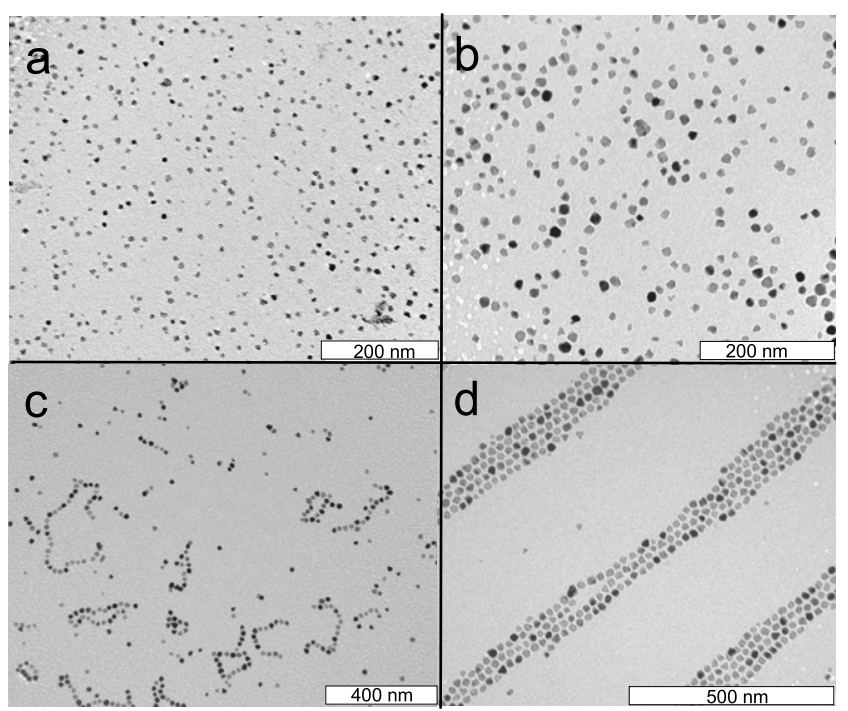

Figure 1. Cryogenic transmission electron microscopy of ferrofluids C (a), B (b), and A (c) in zero field [8, 9]. Panel (d) shows ferrofluid A in a saturating magnetic field of $H=160 \mathrm{kA} \mathrm{m}^{-1}$ [10].

$4 \times 4$ faces oriented parallel to the magnetic field to limit the demagnetization.

A demagnetization factor $D$ of 0.08 was taken into account, calculated on the basis of the prismatic geometry of our sample holders [19]. Demagnetization is significant above $\chi=2$. The error in its calculation is reflected in the error bars in figure 2 and was ascertained experimentally by filling a sample holder with different amounts of ferrofluid. The initial slopes were measured by cycling between -300 and $+300 \mathrm{~A} \mathrm{~m}^{-1}$ in steps of $10 \mathrm{~A} \mathrm{~m}^{-1}$ per s. Full magnetization curves were measured by cycling back and forth to higher maximum fields (e.g., $\pm 25 \mathrm{kA} \mathrm{m}^{-1}$ ) at a scan rate that was sufficiently low to prevent hysteresis ( $25 \mathrm{~A} \mathrm{~m}^{-1} \mathrm{~s}^{-1}$ or lower).

\section{Results}

Figure 1 summarizes our earlier visualization of the dipolar structures in ferrofluids $\mathrm{A}-\mathrm{C}$ by cryogenic transmission electron microscopy [8-10]. This technique provides snapshots of the in situ dipolar structures in two-dimensional ferrofluid films. In ferrofluid $\mathrm{C}$, the dipolar coupling is too weak for dipolar structure formation, in ferrofluid B, zero-field structures start to be visible, and in ferrofluid A, chaining is extensive. In a saturating magnetic field, the dipolar structures are greatly increased in size and the zero-field chains assemble into long, thick columns.

The strength of the dipole-dipole coupling in ferrofluids $\mathrm{A}-\mathrm{C}$ is depicted by the data points in figure 2 , showing the experimentally determined initial magnetic susceptibility as a function of the volume fraction. First, in all three cases, the data exhibit linear behavior at low concentrations. This allows determination of the magnetic coupling constants $\lambda$ (see table 1) using equation (2) (see figure 2, dashed-dotted lines). The linear behavior agrees with the Langevin model, which ignores interparticle interactions and only takes into account the orientational statistics of a single dipole in an external 


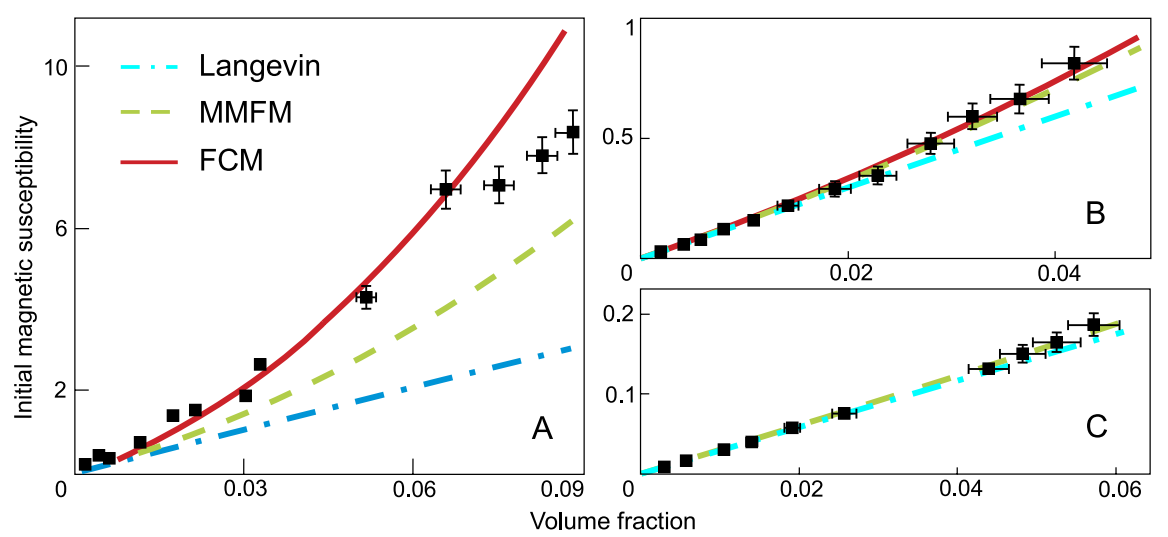

Figure 2. Initial dimensionless magnetic susceptibility $\chi$ versus particle volume fraction $\phi$ for ferrofluids A-C. The theoretical curves through the data points refer to the Langevin equation (equation (2), dashed-dotted line), the modified mean-field model (MMFM, dashed line), and the flexible chain model (FCM, solid line).
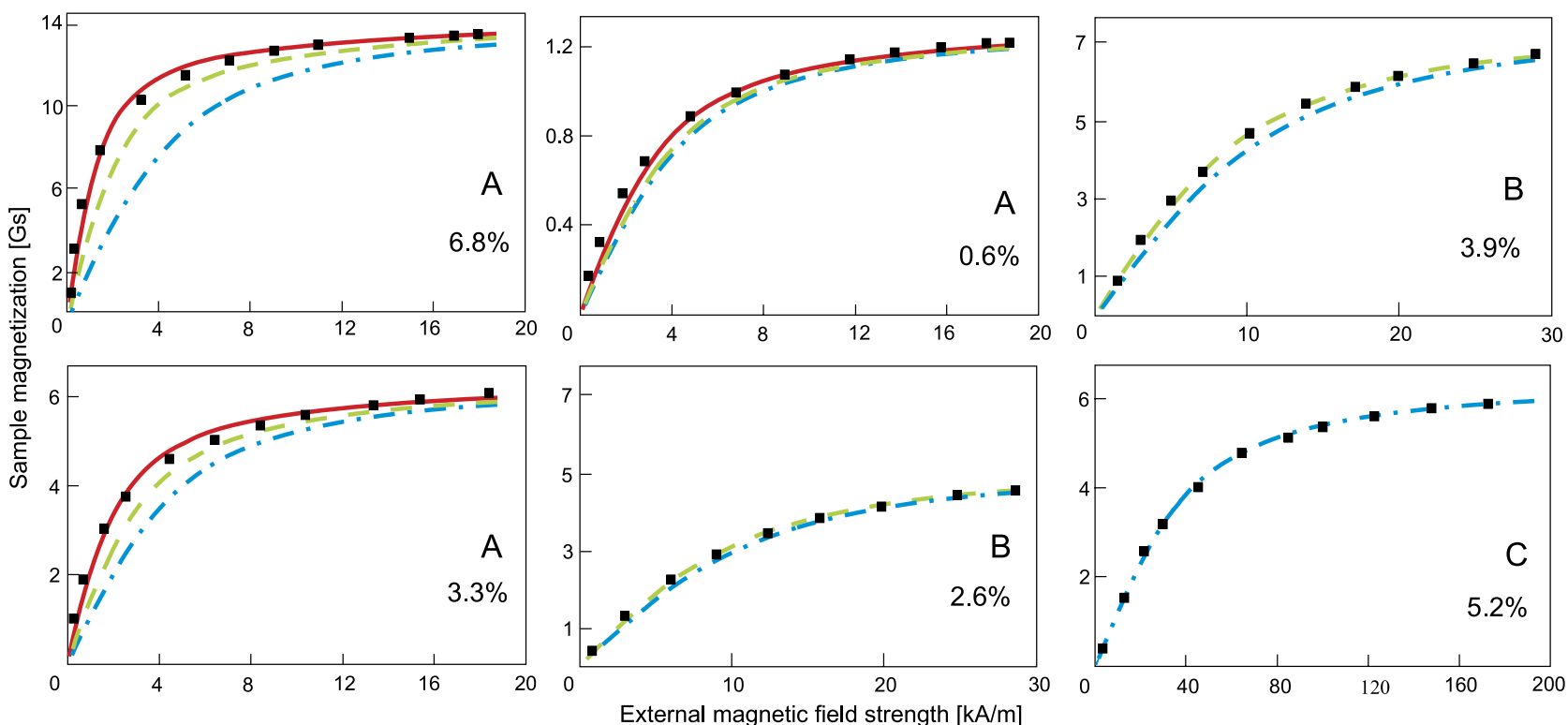

Figure 3. Sample magnetization versus magnetic field strength $\left(\mathrm{kA} \mathrm{m}^{-1}\right)$ for ferrofluids $\mathrm{A}-\mathrm{C}$ at the indicated volume fractions $\phi$. As in figure 2, the dashed-dotted lines are Langevin ones, the dashed lines MMFM ones, and the solid lines flexible chain model ones.

field. Second, at concentrations above $\phi \approx 0.02$, deviations from linearity in figure 2 reveal the effect of interparticle interactions. Due to the strong dipole-dipole coupling $(\lambda=$ 4.4), the deviations are the strongest in system A.

To better highlight the effect of the dipolar structures on the magnetization properties of ferrofluids, we measured the magnetization as a function of the external-field strength for systems $\mathrm{A}-\mathrm{C}$ at different volume fractions $\phi$. Magnetization curves of ferrofluids $\mathrm{A}-\mathrm{C}$ are presented in figure 3 at selected volume fractions $\phi$. For ferrofluid $\mathrm{C}$ (see figure 3, lower right panel), the data correspond to the Langevin equation (equation (1)), as expected in the case of weak dipoledipole interactions. With increasing interactions (system B) small deviations occur at relatively high concentrations $(\phi=$ $3.9 \%)$, indicating moderate but significant interactions $(\lambda=$ 1.7). For strongly dipolar fluids (figure 3 , left panels), the Langevin equation only gives a satisfactory description of the data at very low concentrations $(\phi<0.005)$, where the initial magnetic susceptibility is linear with concentration (figure 2(A)). The strong differences in shape between the experimental data and the Langevin curve at high concentration (figure 3, upper left panel) clearly demonstrate the effect of magnetic dipolar chains on the magnetization properties of a ferrofluid. Since the Langevin approach is no longer applicable for strongly dipolar fluids, two recently developed mean-field approaches that describe dipolar interactions are applied to the experimental data, taking into account the well-defined physical properties of the particles as shown in table 1 (see the previous section).

\section{Discussion}

Until now, the available ferrofluids were highly polydisperse, and a pragmatic approach to testing ferrofluid theory has been to make measurements on concentration series of ferrofluids 
and to extract the average magnetic diameter by modeldependent analysis of the data, the test of theory being that the calculated diameter should be concentration independent [20]. The modified mean-field model of Ivanov and Kuznetsova [21] was the only theory to pass that test when it comes to describing the concentration dependence of the initial magnetic susceptibility of polydisperse ferrofluids that do not contain chain aggregates [22]. However, to describe the field dependence of the magnetization, the model had to be extended to incorporate the field-dependent formation and alignment of flexible dipolar chains $[11,12]$. The measurements in figures 2 and 3 are compared to the modified mean-field model (MMFM) [21] and the flexible chain model (FCM) [11, 12]. Before discussing the results of those comparisons, the main aspects of these models are reviewed.

The development of these and other improved theories for the magnetization of dipolar fluids is done using statistical mechanical perturbation. The MMFM was proposed by Pshenichnikov [23] as a modification of Weiss's mean-field model [2]. The main idea is that the field affecting the orientation of the dipoles consists of the external field $H$ plus a contribution $M_{\mathrm{L}} / 3$ from the surrounding partially aligned dipoles. This leads to an initial susceptibility $\chi=\chi_{\mathrm{L}}(1+$ $\left.\chi_{\mathrm{L}} / 3\right)$. The mean-field models are based on the assumption of a homogeneous distribution of particle positions. However, when the dipole moment is large or the temperature low, the dipole-dipole interaction energy is considerably larger than the thermal energy, and the particles can aggregate even in zero field to form chains (see figure 1). Computer simulations have shown that the particle chains increase the degree of magnetization of ferrofluids in an external field [18, 19]. The simulation data are in qualitative agreement with the model of rigid rod-like chains [24, 25]. However, this corresponds to Langevin orientational statistics of rigid rods, leading to a great overestimation of the chains' response to external fields [25]. In fact, whereas the chain structures are stiff in strong fields, they are flexible in weak fields. This was demonstrated in computer simulations [26, 24] and confirmed by cryogenic transmission electron microscopy [10].

The flexible chain model (FCM) incorporates the MMFM and also accounts for the field-dependent association of dipolar particles into flexible chains. This combined theory was found to be in good agreement with magnetization curves calculated on the basis of molecular dynamics simulations [11, 12]. The FCM expression for the initial magnetic susceptibility is [11]

$$
\chi=\chi_{\mathrm{L}}\left(1+\frac{\chi_{\mathrm{L}}}{3}\right)\left(\frac{1+p_{0} K}{1-p_{0} K}\right)
$$

with

$$
p_{0}=\frac{1+2 q_{0} \phi-\sqrt{1+4 q_{0} \phi}}{2 q_{0} \phi} .
$$

The first term in parentheses corresponds to the mean-field component, and the second term in parentheses corresponds to the flexible chain component. The $p_{0}$ parameter gives the zero-field probability for a magnetic particle to be connected in a chain; $p_{0}$ in combination with the zero-field partition function $q_{0}$ of a dimer determines the chain length distribution. $K$ is the correlation coefficient that describes the orientational rigidity of the chains. Both $q_{0}$ and $K$ depend on the dipoledipole interaction parameter $\lambda$ and on the specific short-range interparticle repulsion $[11,12,22,27]$. For the simplest model of hard sphere dipolar particles, these parameters are given by [11]

$$
q_{0}=\frac{\exp (2 \lambda)}{3 \lambda^{3}}, \quad \text { for } \lambda \gg 1
$$

and

$$
K=L\left(\frac{\lambda}{2}\right)
$$

where $L$ is the Langevin function (equation (1)). The detailed expressions that describe the magnetization as a function of the applied field were presented elsewhere [22, 12, 28, 27]. In the weak field and weak dipole limit, the theory reverts to the Langevin equation. For ferrofluids with a dipolar coupling parameter $\lambda$ below 3 , the mean-field component dominates. Flexible chains manifest themselves above $\lambda \approx$ 3 at concentrations $\phi$ of about $0.01-0.05$, although with very strong dipoles and fields $(\lambda>10$ and $\alpha>10)$, the chains are effectively stiff. Interactions between dipolar chains are neglected in the current flexible chain model but these only become important at (very) high fields where the deviation from the Langevin equation is relatively small. Comparison with computer simulations indicates that the model overestimates the chain length and magnetization above volume fractions of $7 \%[11,12]$.

At weak to moderate dipolar interactions (ferrofluids B and $\mathrm{C}$ ), the flexible chain model $[11,12]$ is difficult to distinguish from the modified mean-field model [21] in the concentration ranges of figure 2. For stronger interactions (ferrofluid A), the initial magnetic susceptibility data appear to correspond to the flexible chain model up to concentration of $7 \%$. Conclusive are the magnetization curves of ferrofluid A in figure 3 , which clearly are well described by the flexible chain model in the concentration regime $0-7 \%$. The deviations observed at concentrations $>7 \%$ are likely to be related to inter-chain interactions. All these observations agree with the well-known trend that extensive dipolar chaining of magnetic particles requires both a high coupling parameter $\lambda$ and a high concentration $\phi$.

\section{Conclusion}

In conclusion, we have measured the field-dependent magnetization of model ferrofluids for which dipolar chaining was demonstrated independently using cryogenic transmission electron microscopy. The switch to extensive dipolar chaining that occurs when the concentration and particle dipole moment become large enough is accompanied by a switch in the magnetic behavior. Without chains, the initial susceptibility is linear with concentration and the magnetization curve is described by the Langevin equation (equation (1)). With chains, the concentration dependences of the initial susceptibility and the magnetization curves agree with the theory that accounts for the magnetic effects of fieldinduced dipolar chaining $[11,12]$. As a bonus, the dipolar chains that affect the magnetic properties have been imaged in situ by cryogenic electron microscopy, making it even more 
convincing that the effects ascribed in theory and simulations to the field-dependent formation and alignment of dipolar chains match the behavior in actual experiments.

\section{Acknowledgments}

This work was partially supported by the 'Dynasty' Foundation, INTAS Grant No 03-51-6064. We thank C Holm, S Kantorovich, and A P Philipse for helpful discussions.

\section{References}

[1] Langevin P 1905 J. Physique 4678

[2] Weiss P 1907 J. Physique Rad. 4661

[3] Debye P 1912 Z. Phys. 1397

[4] Onsager L 1936 J. Am. Chem. Soc. 581486

[5] Huke B and Lücke M 2004 Rep. Prog. Phys. 671731

[6] van Ewijk G, Vroege G and Philipse A 2002 J. Phys.: Condens. Matter 144915

[7] Charles S 1988 Chem. Eng. Commun. 67145

[8] Klokkenburg M, Vonk C, Claesson E, Meeldijk J, Erné B and Philipse A 2004 J. Am. Chem. Soc. 12616706

[9] Klokkenburg M, Dullens R, Kegel W, Erné B and Philipse A 2006 Phys. Rev. Lett. 96037203
[10] Klokkenburg M, Erné B, Meeldijk J, Wiedenmann A, Petukhov A, Dullens R and Philipse A 2006 Phys. Rev. Lett. 97185702

[11] Mendelev V and Ivanov A 2004 Phys. Rev. E 70051502

[12] Mendelev V and Ivanov A 2005 J. Magn. Magn. Mater. 289211

[13] Klokkenburg M and Erné B 2005 J. Magn. Magn. Mater. 30685

[14] Klokkenburg M, Hilhorst J and Erné B 2007 Vib. Spectrosc. 43243

[15] Berkovski B and Bashtovoy V 1996 Magnetic Fluids and Applications Handbook (New York: Begel House)

[16] Kodama R 1999 J. Magn. Magn. Mater. 200359

[17] Flanders P 1988 J. Appl. Phys. 633940

[18] Harrell J 1999 J. Magn. Magn. Mater. 205121

[19] Aharoni A 1998 J. Appl. Phys. 833432

[20] Raşa M, Bica D, Philipse A and Vékás L 2002 Eur. Phys. J. E 7209

[21] Ivanov A and Kuznetsova O 2001 Phys. Rev. E 64041405

[22] Ivanov A and Kuznetsova O 2006 Colloid J. 68430

[23] Pshenichnikov A 1995 J. Magn. Magn. Mater. 145319

[24] Ivanov A and Kantorovich S 2004 Phys. Rev. E 70021401

[25] Zubarev A and Iskakova L 1995 J. Exp. Theor. Phys. 80857

[26] Wang Z, Holm C and Müller H 2002 Phys. Rev. E 66021405

[27] Holm C, Ivanov A, Kantorovich S, Pyanzina E and Reznikov E 2006 J. Phys.: Condens. Matter 18 S2737

[28] Ivanov A, Kantorovich S, Mendelev V and Pyanzina E 2006 J. Magn. Magn. Mater. 300 e206 